\title{
Ernährungstherapie des Metabolischen Syndroms
}

Das Metabolische Syndrom ist definiert durch das sogenannte tödliche Quartett, d.h. abdominelle Adipositas, Dyslipoproteinämie, Typ-2-Diabetes und Hypertonie. Die gemeinsame Grundlage dieses Syndroms besteht in der Insulinresistenz, worunter eine reduzierte Insulinwirkung mit kompensatorisch erhöhten Insulinspiegeln verstanden wird. Bei Progredienz dieser Störung in Richtung Typ-2-Diabetes entwickelt sich zunehmend eine verzögerte und später ausbleibende

Mit der LOGI-Diät und Insulinausschüttung nach GlukoOmega-3-Fettsåuren spiegel ständig erhöht sind(Sekregegen Insulinresistenz tionsstarre). Als Konsequenz entstehen hohe postprandiale

Blutzuckerwerte nach kohlenhydratreichen Mahlzeiten sowie die Neigung zu Hypoglykämien mit Heißhungerattacken bei fehlendem Kohlenhydratnachschub. Gleichzeitig fällt die Hemmwirkung auf die Glukoneogenese weg (ungebremste Glukoneogenese). Die gestörte Glukosetoleranz ist bereits frühzeitig in der Entwicklung des Metabolischen Syndroms nachweisbar. Die in Folge zu hoher postprandialer Blutzuckerwerte entstehenden Advanced Glycosilation Endproducts (AGE) gelten heute als wesentlicher Faktor für die Entstehung der Zellschädigung im Rahmen des Metabolischen Syndroms.

Die Ernährungstherapie des Metabolischen Syndroms mit und ohne Typ-2-Diabetes war über Jahrzehnte durch die Empfehlung einer fettreduzierten kohlenhydratreichen Kost geprägt, da man den Fettgehalt der Nahrung als Hauptursache der Arteriosklerose betrachtete (sogenannte Lipotoxizität). Noch 2005 empfahl die Deutsche Diabetes-Gesellschaft eine Diät mit einem Kohlenhydratanteil von bis zu $60 \%$. Dieser hohe Kohlenhydratgehalt führt aber zu entsprechend starker Belastung der Betazellen, wodurch deren Versagen beschleunigt wird. Das Entstehen postprandialer Blutzuckerspitzen lässt sich pharmakologisch nur teilweise kompensieren. Eine weitere ungünstige Wirkung der fettreduzierten Kost ist die Senkung des gefäßprotektiven HDL-Cholesterins.
Eine Alternative der Ernährungsempfehlung mit reduziertem Kohlenhydratanteil (auf ca. 25-30\%) und einer deutlichen Steigerung des Fett- und Eiweißgehaltes (auf 45-50 bzw. 30\%) wurde von David Ludwig (Harvard-University Boston) erfolgreich bei Jugendlichen mit Metabolischem Syndrom eingesetzt. Diese LOGI-Diät (Low Glycemic and Insulinemic Nutrition) wurde von Nicolai Worm in Deutschland eingeführt. Bei der Reduktion der Kohlenhydrate sollten vor allem kohlenhydratreiche Lebensmittel mit hohem glykämischen Index weitgehend vermieden werden. Ferner sollte im Rahmen der Steigerung der Fettzufuhr darauf geachtet werden, dass der Anteil entzündungshemmender Omega-3-Fette deutlich erhöht wird. Wir setzen seit drei Jahren an unserer Klinik die LOGI-Diät bei Typ-2-Diabetikern ein. Dabei fanden wir eine erhebliche Verbesserung des Blutzuckerprofils, sodass 76\% der antidiabetischen Medikation abgesetzt werden konnte. Ferner ergab sich ein Wegfall postprandialer Blutzuckerspitzen sowie eine erhebliche Verbesserung der Dyslipoproteinämie mit Absenkung der Triglyceride um über $30 \%$ und Senkung von Cholesterin und LDL um mehr als $10 \%$. Trotz ad-libidum-Verzehr nahmen unsere Patienten im Durchschnitt ca. 1 kg pro Woche ab. In einer noch nicht veröffentlichten Studie zum Fettsäureprofil ergaben sich hoch signifikante Veränderungen zu Gunsten der herzschützenden Omega-3-Fettsäuren. Angesichts dieser sehr ermutigenden Ergebnisse sollten die bisherigen Diätempfehlungen dringend überdacht und vergleichende Ernährungsstudien mit harten Endpunkten (KHK-Morbidität, Gesamtmortalität) durchgeführt werden.

\author{
Dr. med. Peter Heilmeyer \\ Leitender Arzt \\ Reha-Klinik Überruh \\ 88316 Isny/Allgäu \\ p.heilmeyer@rehaklinik-ueberruh.de
}

